\title{
Menores quemados por el sol y su relación con la radiación ultravioleta y la cubierta de ozono, durante seis veranos (1996 a 2001) en Santiago de Chile $\left(33,5^{\circ} \mathrm{S}\right)$
}

\author{
Ligia Aranibar $\mathrm{D}^{1}$, Sergio Cabrera $\mathrm{S}^{2}$, Juan Honeyman $\mathbf{M}^{3}$. \\ Association between sunburns \\ in children and ultraviolet radiation \\ during summer in Santiago, Chile
}

Background. During the recent 10 years the ozone layer has decreased while ultraviolet radiation has increased in Santiago, Chile. Aim: To determine whether the number of sunburns in children correlate with ultraviolet radiation in Santiago. Subjects and methods: During six Austral Summers (1996-2001) children below 15 years old, consulting for sunburn, were evaluated at the "Corporation for the Aid of Burned Children" (COANIQUEM) in Santiago (33.5 ${ }^{\circ}$ ). The number of children with sunburns during each Summer was compared with the corresponding UV-B radiation and the ozone thickness, to establish a probable relation between a geophysical change and its consequences in skin health. The ozone layer values were obtained from the NASA WEB-page and the ultraviolet radiation was measured with a four-channel medium resolution radiometer. Results: In each Summer there was a predominance of sunburns among boys and among ages between 6 and 10 years. During the 96-97 Austral Summer, the highest number of children with sunburns (63) was diagnosed. That Summer also had the highest mean UV-305 nm radiation with an important amount of days with ozone $\leq 260$ Dobson Units. Only during that Summer an inverse correlation between ozone and UV-305 nm radiation was detected. At the same time the maximal values of Erythemal Dose Rate $\left(33 \mu\right.$ Watt $\left.\mathrm{cm}^{-2}\right)$, UV Index (13) and Erythemal Daily Dose (7.500 Joule $\mathrm{m}^{-2}$ ) were observed. Conclusions: In Santiago, Summers with a higher number of days with low ozone protection seem to reappear every 3 years. Understanding the interaction of physical processes that control the ozone layer, may help to design better photoprotection programs for human health (Rev Méd Chile 2003; 131: 1011-22).

(Key Words: Ozone; Sunburn; Ultraviolet rays; UV-Index)

\footnotetext{
Recibido el 8 de abril, 2003. Aceptado en versión corregida el 11 de agosto, 2003.

Fuente de apoyo financiero: Proyecto IAI "InterAmerican Institute" Global Change Research CNR 026 y Departamento de Investigacion y Desarrollo (DID) de la Universidad de Chile.

${ }^{1}$ Fundación de Ayuda al Niño Quemado y Departamento de Dermatología, Hospital Clínico de la Universidad de Chile; actualmente Unidad de Dermatología Occidente y Servicio de Dermatología del Hospital San Juan de Dios.

2 Profesor de Biología y Ciencias (Fotobiología), Profesor Titular, Instituto de Ciencias Biomédicas Programa de Biología Celular y Molecular, Facultad de Medicina, Universidad de Chile.

${ }^{3}$ Departamento de Dermatología, Hospital Clínico Universidad de Chile.
}

Correspondencia a: Prof. Sergio Cabrera. Casilla 70061-Correo 7. Santiago.

E-mail: scabrera@machi.med.uchile.cl 
$\mathrm{E}^{\mathrm{s}}$ n los últimos 30 años, se ha producido disminución del ozono $\left(\mathrm{O}_{3}\right)$ estratosférico en las primaveras de ambos hemisferios, y se ha constatado disminución global de $\mathrm{O}_{3}$ en latitudes medias de $0,8 \% 1$ y de $1,4 \%$ anual $^{2}$ en Santiago.

Esto ha traído como consecuencia que ciudades como Santiago y Buenos Aires, reciban mayor radiación UV-B (315-280 nm). Aunque es posible afirmar que el $\mathrm{O}_{3}$ absorbe una ínfima porción de radiación UV-A (315-400 nm), el UV-B es más eritematogénico ${ }^{3}$, y es el que se incrementa cuando disminuye el $\mathrm{O}_{3}$.

La cantidad de radiación UV-B que llega a la superficie terrestre depende fundamentalmente: 1) del ángulo cenital del sol, 2) del $\mathrm{O}_{3}$ estratosférico y troposférico existente, 3) de las moléculas y partículas componentes de la atmósfera (dispersión), y 4) de la ubicación geográfica (latitud y altura) $)^{4}$.

El aumento de UV-B en la población, tiene efectos agudos y crónicos. Los agudos son: la quemadura solar y la fototoxicidad inducida, si hay ingesta de fármacos; y los crónicos pueden provocar cambios como: envejecimiento prematuro, adelgazamiento irregular de la epidermis, telangiectasias, léntigos, queratosis actínicas, carcinoma espino celular, carcinoma baso celular y melanoma.

El objetivo de este trabajo es relacionar el número de niños (menores de 15 años) quemados por el sol que solicitaron atención en la Corporación de Ayuda al Niño Quemado (COANIQUEM) entre los veranos 1995/96 y 2000/01, con el espesor de ozono estratosférico y la radiación UVB que recibió Santiago.

\section{MATERIAL y MÉTOdo}

1) Clínica. En COANIQUEM, desde 1995 uno de los autores (LA) implementó un protocolo clínico sistemático para niños quemados por sol, que incluye: nombre, edad, sexo, domicilio, antecedentes de medicamentos o enfermedades fotosensibles, tiempo de exposición, uso de protector solar, lugar de la quemadura y tipo de piel ${ }^{5}$.

La quemadura solar se define por la presencia de eritema de la piel producido por vasodilatación de los capilares dérmicos, que se presenta entre 12 y 24 h después de la exposición. Esta manifes- tación se acompaña de calor local y sensación de ardor. En casos más severos se originan vesículas y ampollas dolorosas.

El diagnóstico de quemadura solar se realizó mediante la anamnesis y examen físico realizado por el personal de enfermería capacitado para este tipo de patología y corroborado por un médico.

Para cada verano, se calculó el número de quemados por cada mil consultas, y se aplicó la prueba de Chi-cuadrado entre el número de quemados por sol y el número de quemados por los otros agentes, para comprobar su independencia. Se dividió la muestra por sexo y edades (0-5; 6-10 y 11-15 años).

2) Radiación UV y parámetros fotobiológicos. Los valores de radiación UV se miden en forma permanente y continua desde enero de 1992 a la fecha, en el Instituto de Ciencias Biomédicas de la Facultad de Medicina de la Universidad de Chile, con un equipo de banda media fabricado por Biopherical Instruments Inc. San Diego, California, con cuatro canales centrados en 305, 320, 340 y 380 nanómetros (nm). Los valores entre 1992-1998 se estandarizaron a $20^{\circ} \mathrm{C}$ y el radiómetro se calibró 5 veces contra un equipo de referencia en el período.

Para comparar las estaciones de verano entre sí, se utilizó el valor promedio y desviación estándar $(\sigma)$ de las mediciones de UV-305 nm. En adelante la sigla UV-B se empleará para referirse al canal de $305 \mathrm{~nm}$.

Se entenderá como verano, al período entre el 7 de noviembre y 5 de febrero del año siguiente, centrado en el solsticio de verano (22 de diciembre).

Para recuperar el espectro UV (300-400 nm), a partir de las mediciones en 4 canales, se utilizó un método de inversión matemática ${ }^{6}$.

Para estimar la Tasa de Dosis (TD) y Dosis Diaria de Eritema (DDE) se emplearon los métodos propuestos por Madronich ${ }^{7}$.

La TD es la forma de dimensionar el efecto que produce el conjunto de fotones ultravioleta que recibe el hombre en un instante de tiempo, y se calcula ponderando las irradianzas en las distintas longitudes de onda según su efectividad para producir una respuesta (Espectro de Acción de Eritema); se expresa en $\mu \mathrm{W} \mathrm{cm}^{-2}$. 
La DDE es equivalente a la suma de las TD, desde la salida a la puesta del sol; se expresa en Joule $/ \mathrm{m}^{2}$.

El índice UV, es un valor adimensional y equivale al múltiplo 40 de la TD expresada en $\mathrm{W} / \mathrm{m}^{2}$. Valores típicos del índice UV, van de 0 a 10, pero existen valores superiores.

Para estudiar las variaciones interanuales, se analizó el promedio y tendencia de los 9 días de inicio y 9 finales del verano; ellos comparten igual ángulo cenital del sol. El período inicial va del $7 \mathrm{al}$ 16 de noviembre y el de término, va del 27 de enero al 5 de febrero.

Cubierta de ozono. Para conocer las variaciones interdiarias y para observar el área geográfica que cubren las masas de aire con bajo $\mathrm{O}_{3}$, se utilizó la página web de la National Aeronautic and Space Administration (NASA) (http://jwocky.gsfc.nasa.gov) desde donde se obtuvieron los valores del $\mathrm{O}_{3}$ total. Estos datos han sido corregidos para simular el paso del satélite sobre Santiago. El $\mathrm{O}_{3}$ de la columna de aire, se expresa en Unidad Dobson (UD) (300 UD equivalen a $3 \mathrm{~mm}$ de espesor, cuando el $\mathrm{O}_{3}$ es sometido a 1 atmósfera de presión).

No hay mediciones de $\mathrm{O}_{3}$ desde el satélite (24 de noviembre de 1994 y el 25 de julio de 1996).

Debido a que el mayor número de niños quemados por sol se produjo entre el 11 de diciembre y 5 de febrero, se comparó la significación estadística, entre los valores promedios de $\mathrm{O}_{3}$ de este período denominado "verano breve".

\section{RESULTADOs}

Antecedentes clínicos. Durante estos 6 veranos el número de niños quemados por el sol varió sin presentar una tendencia clara. El número fluctuó entre 13 y 63, siendo el verano 96/97 el que presentó el mayor registro, seguido del 99-00. La estimación de quemados/1.000 atendidos en ambos veranos tiene un valor cercano a 40 , en cambio, en los otros veranos fue 20 ó menor (Figura 1a).

Al comparar el número de niños quemados por el sol, con aquellos quemados por otros agentes, se obtiene que son significativamente diferentes, con una probabilidad inferior a $0,005 \%$.

Los varones generalmente duplican en número a las mujeres, con las excepciones de los verano
96-97 y 00-01 cuando se presentaron en número similar (Figura 1b).

Durante los 6 veranos predominó el grupo etario de 6 a 10 años; pero llama la atención que en 3 oportunidades (96-97, 97-98, y 99-00), el grupo de 0 a 5 años sobrepasa el $50 \%$ del grupo de 6 a 10 años (Figura 1c).

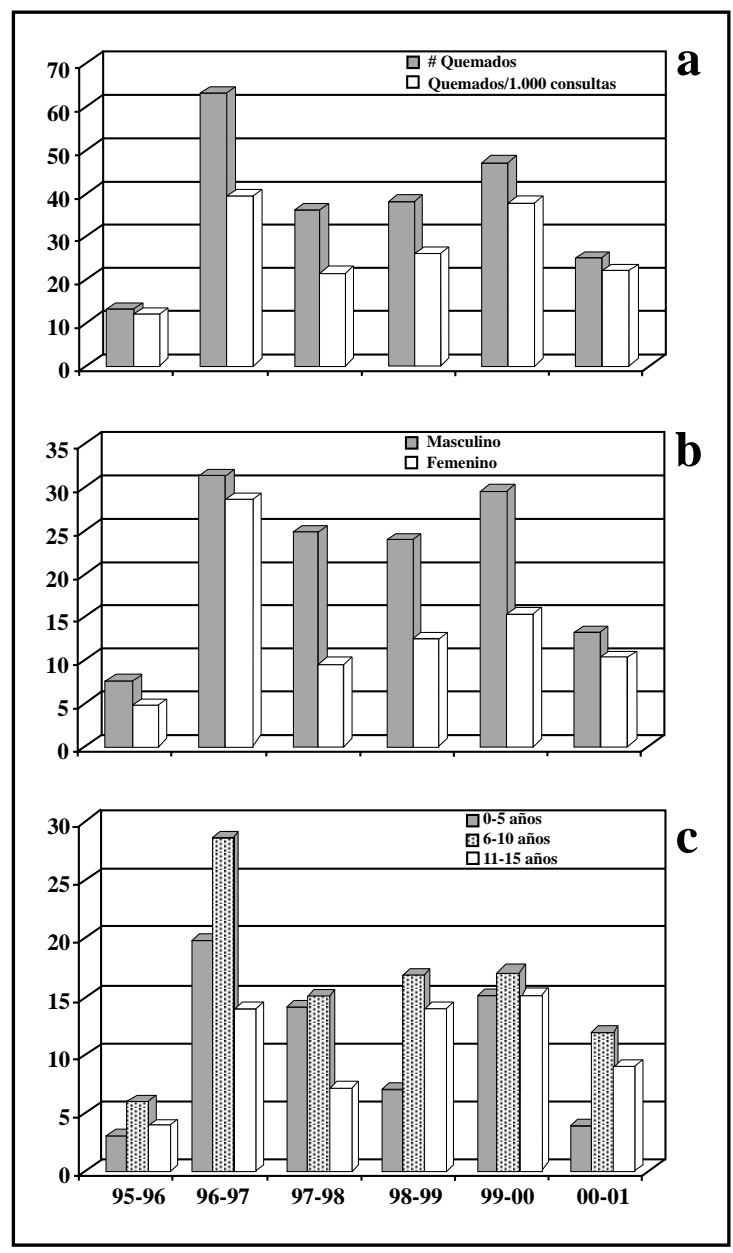

Figura 1. En los gráficos a, b y c, el eje horizontal representa los veranos incluidos en esta presentación. 1a. Las barras grises representan el número de niños quemados por sol y las barras blancas el número de niños quemados por el sol/1.000 consultas en cada verano. 1b. Distribución por sexo de los niños quemados por sol. Barras grises varones y barras blancas damas. 1c. Distribución etaria de los niños quemados por sol. 


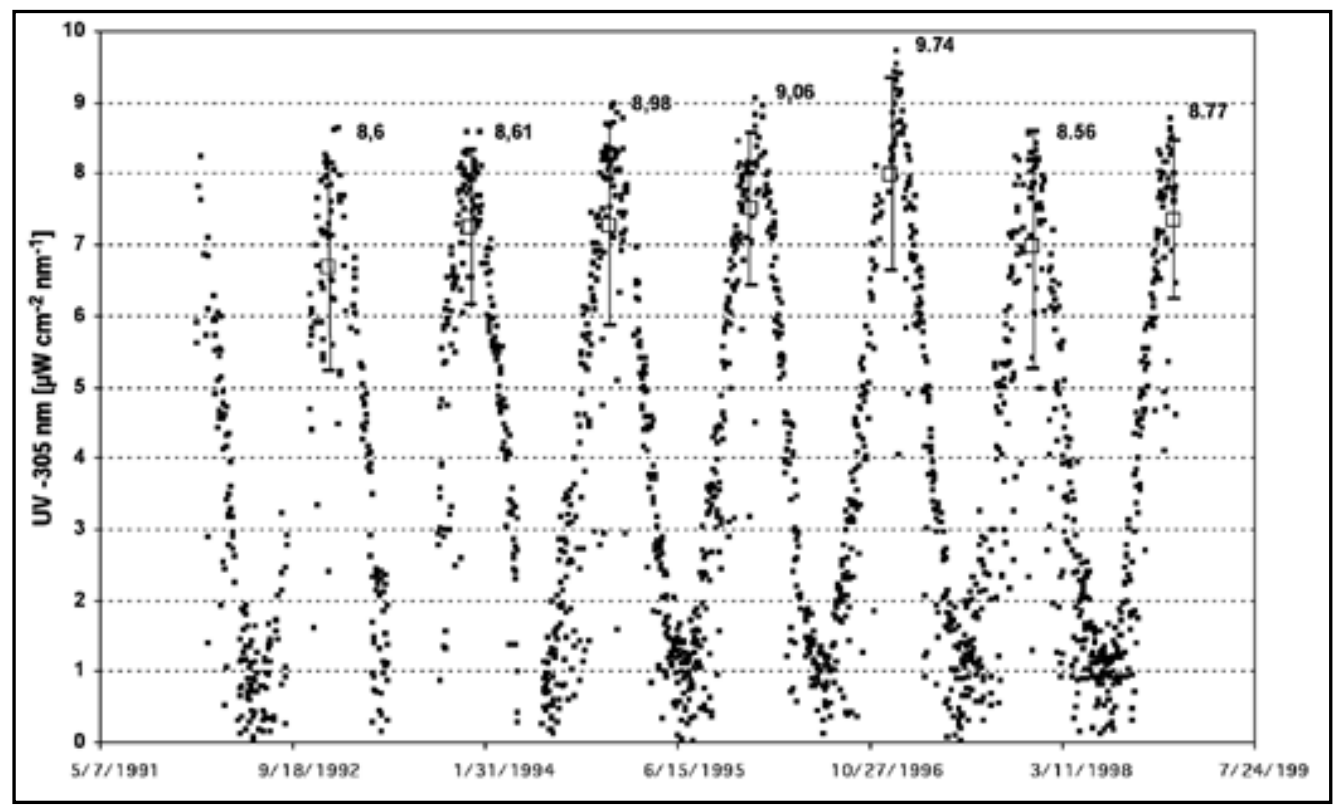

Figura 2. Valores promedios horarios alrededor del mediodía solar de radiación UV-B. El cuadrado representa el promedio del verano y las líneas verticales una desviación estándar. La cifra indicada en cada verano corresponde al valor máximo alcanzado en la temporada.

\section{Antecedentes geofísicos}

Radiación ultravioleta $305 \mathrm{~nm}$ (UV-B). En la Figura 2, la radiación UV-B fluctúa de cero a 8-9 $\mu \mathrm{W} \mathrm{cm}^{-2} \mathrm{~nm}^{-1}$, desde invierno a verano.

El análisis de varianza de los promedios de la radiación UV-B de los veranos analizados indica, que todos son significativamente diferentes al verano 96-97 con una probabilidad inferior a 5\%.

Los promedios de la radiación UV-B de los 9 días de inicio del verano fueron menores que el de los 9 días de término (excepto el 97-98). Esta diferencia debe atribuirse a la mayor cubierta de $\mathrm{O}_{3}$ al inicio del verano, pues ambos períodos tienen igual ángulo cenital. A partir del 92-93, los promedios del período inicial fueron sistemáticamente crecientes (Tabla 1).

La regresión lineal entre el promedio estacional de UV-B en el período 92-98 presentó pendiente positiva que no es significativa (Figura 3 ).

La regresión lineal del promedio de UV-B de los 9 días iniciales tuvo pendiente positiva significativa al 1\%. Se omitió el 92-93, por el escaso número de datos (Figura 3). Si se hubiera mantenido la pendiente de la regresión, los promedios de los 9 días iniciales para 01-02 y 02-03 deberían haber sido cercanos a 8,073 y $8,371 \mu \mathrm{W} \mathrm{cm}^{-2} \mathrm{~nm}^{-1}$, respectivamente.

La regresión lineal del promedio de UV-B de los 9 días finales también presentó una pendiente positiva, no significativa (Figura 3).

Tabla 1. Promedio de radiación UV-B al inicio y término del verano

\begin{tabular}{|lcr|}
\multicolumn{2}{c}{$\left[\mu \mathrm{W} \mathrm{cm}^{-2} \mathrm{~nm}^{-1}\right]$} \\
\hline & $\begin{array}{c}\text { 9 días inicio } \\
\text { verano (n) }\end{array}$ & $\begin{array}{c}\text { 9 días término } \\
\text { verano (n) }\end{array}$ \\
\hline $92 / 93$ & $4,021(3)$ & $6,9696(5)$ \\
$93 / 94$ & $5,885(9)$ & $6,7825(2)$ \\
$94 / 95$ & $6,176(9)$ & $7,0567(7)$ \\
$95 / 96$ & $6,419(9)$ & $7,9448(6)$ \\
$96 / 97$ & $6,674(8)$ & $7,4498(9)$ \\
$97 / 98$ & $6,991(9)$ & $4,1082(4)$ \\
$98 / 99$ & $7,011(9)$ & $(0)$ \\
\hline
\end{tabular}

( ) \# de días con registro de radiación UV-B. 


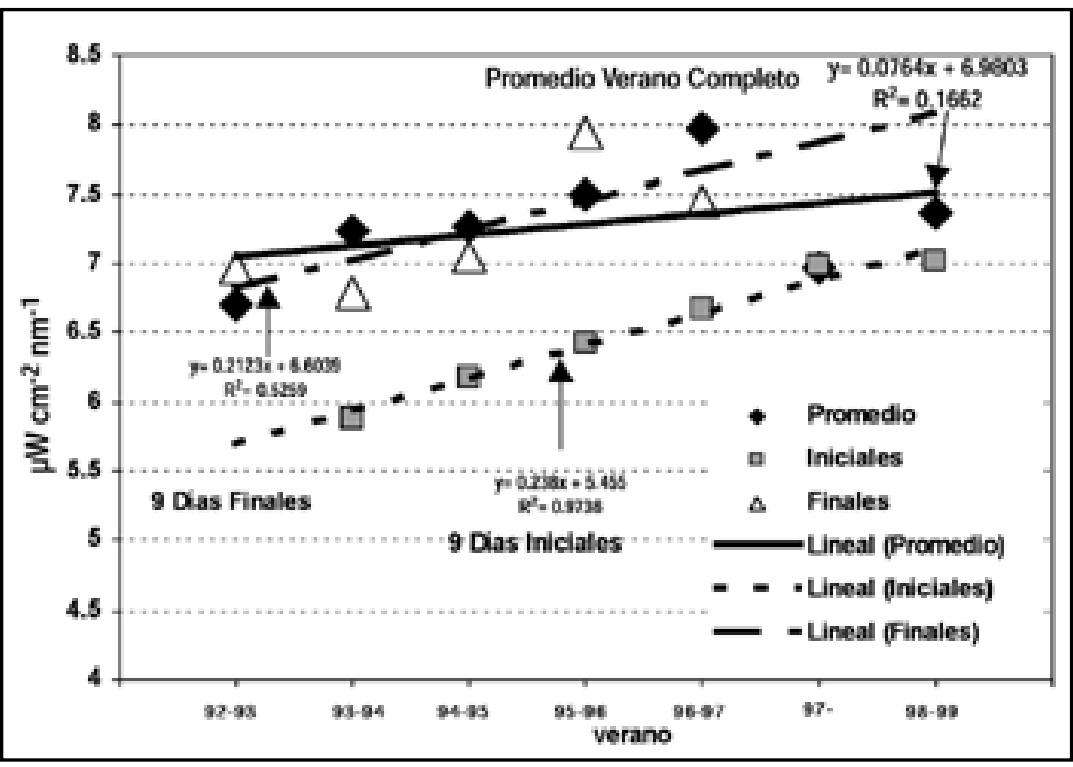

Figura 3. Línea de regresión entre los valores estacionales de radiación UV-B (valores promedios horarios alrededor del medio solar), triángulos abiertos - línea continua. Línea de regresión entre los valores promedios de los 9 días iniciales del verano de la radiación UV-B (valores promedios horarios alrededor del mediodía solar), cuadrados grises - línea segmentada. Ĺnea de regresión entre los valores promedios de los 9 días finales del verano de la radiación UV-B (valores promedios horarios alrededor del mediodía solar), rombos negros - línea de segmentos cortos y largos.

Cubierta de ozono. En la Figura 4a el promedio decadal de $\mathrm{O}_{3}$ (79-88) disminuyó con pendiente constante entre el 7 de noviembre y el 31 de diciembre; pasando de 320 a 280 UD. Desde el inicio del año hasta el 28 de febrero, el $\mathrm{O}_{3}$ se estabilizó alrededor de 280 UD. Si bien, el $\mathrm{O}_{3}$ en este último período se mantuvo estable, el ángulo cenital del sol aumentó.

En la Figura 4b, se compara la cubierta de $\mathrm{O}_{3}$ histórica con las aquí analizadas (96-97 al 00-01). Se observa que en la primera mitad del verano (07 de noviembre al 22 de diciembre) no presentan diferencias marcadas. Sin embargo, posterior al solsticio, desde el 23 de diciembre al 5 de febrero, la mayor parte de estos veranos presentaron valores menores al promedio histórico.

El promedio histórico de $\mathrm{O}_{3}$ del verano es $288,28 \pm 8,96$ UD, y esta cifra es $3 \%$ superior al promedio de $\mathrm{O}_{3}$ de 96-97, aumentando al 5\% en el verano breve. En la Figura $4 \mathrm{~b}$ se ha trazado una línea en 260 UD, para destacar el número de días con valores menores indicados en la Tabla 2 .

Los veranos completos y los "breves" comparados con su referente histórico fueron estadísticamente diferentes $(p=0,001)$.

Relación número de quemados, radiación UV-B y cubierta de $\mathrm{O}_{3}$ En la Figura 5a, se presenta el número de niños quemados por sol que se registra- ron en COANIQUEM, el mismo día o posterior a los momentos con bajo $\mathrm{O}_{3}$.

En los veranos posteriores a 96-97 no se observó la anticorrelación entre $\mathrm{O}_{3}$ y UV-B como se ve en la Figura 5a; en estos veranos la radiación UV-B se presentó como una línea horizontal levemente fluctuante alrededor de los valores $7 \mathrm{y}$ $8 \mu \mathrm{W} \mathrm{cm}^{-2} \mathrm{~nm}^{-1} \mathrm{y}$ sin la suave forma de campana observada en el verano 96-97 (Figura 5a).

De la Tabla 2 se ve un ciclo, en que al tercer verano aumenta el número de días con bajo $\mathrm{O}_{3}$ (96-97 y 99-00) los que coinciden con los veranos con mayor número de niños quemados por sol.

Parámetros fotobiológicos. TD, DDE e índice UV. En la Figura 5b, se observa que la TD, desde el 20 de diciembre (96-97) se separa de los otros dos veranos (anterior y posterior) para nuevamente unirse a éstos alrededor del 20 de enero. Este período de mayor radiación UV-B es coincidente con la menor cubierta de $\mathrm{O}_{3}$ (Figura 5a) e igual momento en donde se observa el quiebre de la pendiente en la Figura $4 \mathrm{~b}$.

El índice UV, entre el 25 de diciembre 1996 y 15 de enero 1997, fluctuó entre 12 y 13 con un máximo de 13,3. A partir de 11 se consideran "extremos" en la calificación de la Organización Mundial de la Salud (OMS). La OMS, considera muy altos los índices UV 8 a 10 y altos entre 6 y 7 . 


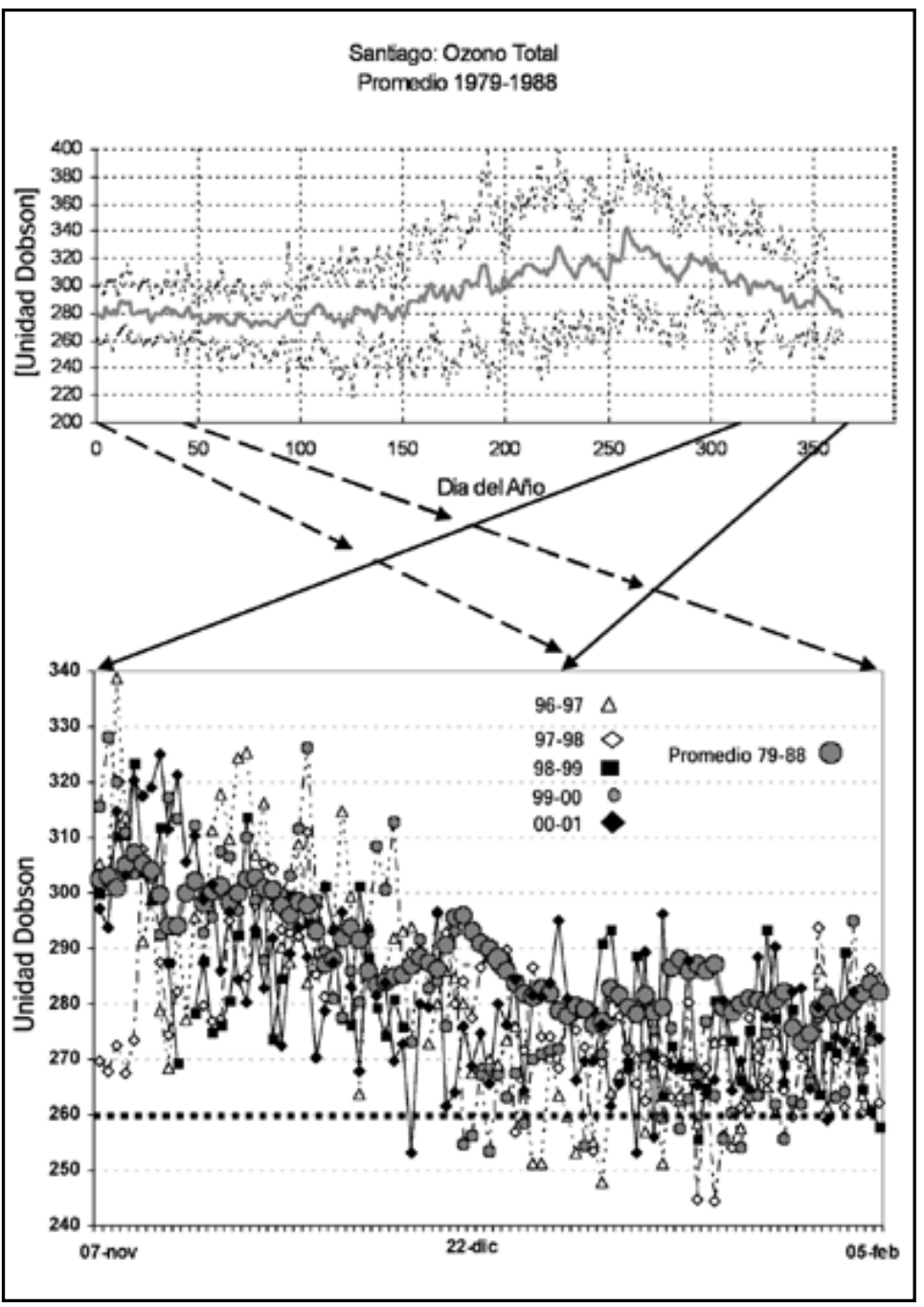

Figura 4a. Promedio diario histórico (línea negra) de diez años $\pm 2 \sigma$ (línea discontinua delgada) del espesor de la capa de ozono total sobre Santiago, expresado en Unidades Dobson, y medido desde el equipo TOMS, instalado en un satélite.

Figura 4b. Promedio diario del ozono histórico de la década (79-88) versus el ozono diario de los veranos 97-98 al 00-01, expresado en Unidades Dobson.

Tabla 2. 0 zono $\left(\mathrm{O}_{3}\right)$ [Unidad D obson UD ]

\begin{tabular}{|lccccc|}
\hline Verano & $\begin{array}{c}\text { Verano completo } \\
\text { 07 nov a 05 feb }\end{array}$ & $\mathrm{n}=$ día & $\begin{array}{c}\text { Verano breve } \\
11 \text { dic a 05 feb }\end{array}$ & $\mathrm{n}=$ día & días 03 $\leq 260$ UD \\
\hline $\begin{array}{l}\text { Década } \\
79-88\end{array}$ & $288,28 \pm 8,9 *$ & 91 & $282,8 \pm 5,0$ & 56 & \\
histórico & & & & & \\
$96-97$ & $280,5 \pm 20$ & 84 & $268,7 \pm 11$ & 51 & 12 \\
$97-98$ & $276,98 \pm 14$ & 78 & $272,8 \pm 12$ & 54 & 7 \\
$98-99$ & $282,8 \pm 15$ & 68 & $273,9 \pm 9,6$ & 34 & $3^{* *}$ \\
$99-00$ & $282,3 \pm 19$ & 89 & $270,8 \pm 11,8$ & 56 & 10 \\
$00-01$ & $281,8 \pm 16$ & 90 & $273,7 \pm 10,3$ & 56 & 4 \\
\hline
\end{tabular}

*Promedio \pm Desviación estándar. *22 días sin medición $\mathrm{O}_{3}$ de 56 . 
Figura 5a. Relación entre cubierta de Ozono, radiación ultravioleta y número de niños quemados registrados en COANIQUEM en el verano 96-97. Rombos oscuros indican ozono (eje vertical izquierdo), los cuadrados grises corresponden al promedio horario de radiación UV-B (eje vertical derecho) y los triángulos abiertos representan el número de niños quemados por sol (eje vertical derecho).

Figura 5b. TD, DDE e índice UV. Se presentan los valores de la tasa de dosis, de los valores promedios horarios alrededor del mediodía solar, del período de verano breve (7 diciembre al 5 de febrero) correspondientes a los años (95-96; 96-97 y 97-98). El que presentó el mayor flujo de radiación UV-B, TD e índice UV fue 96-97 (círculos grises), y se compara con igual período del verano anterior (95-96) y posterior (97-98).

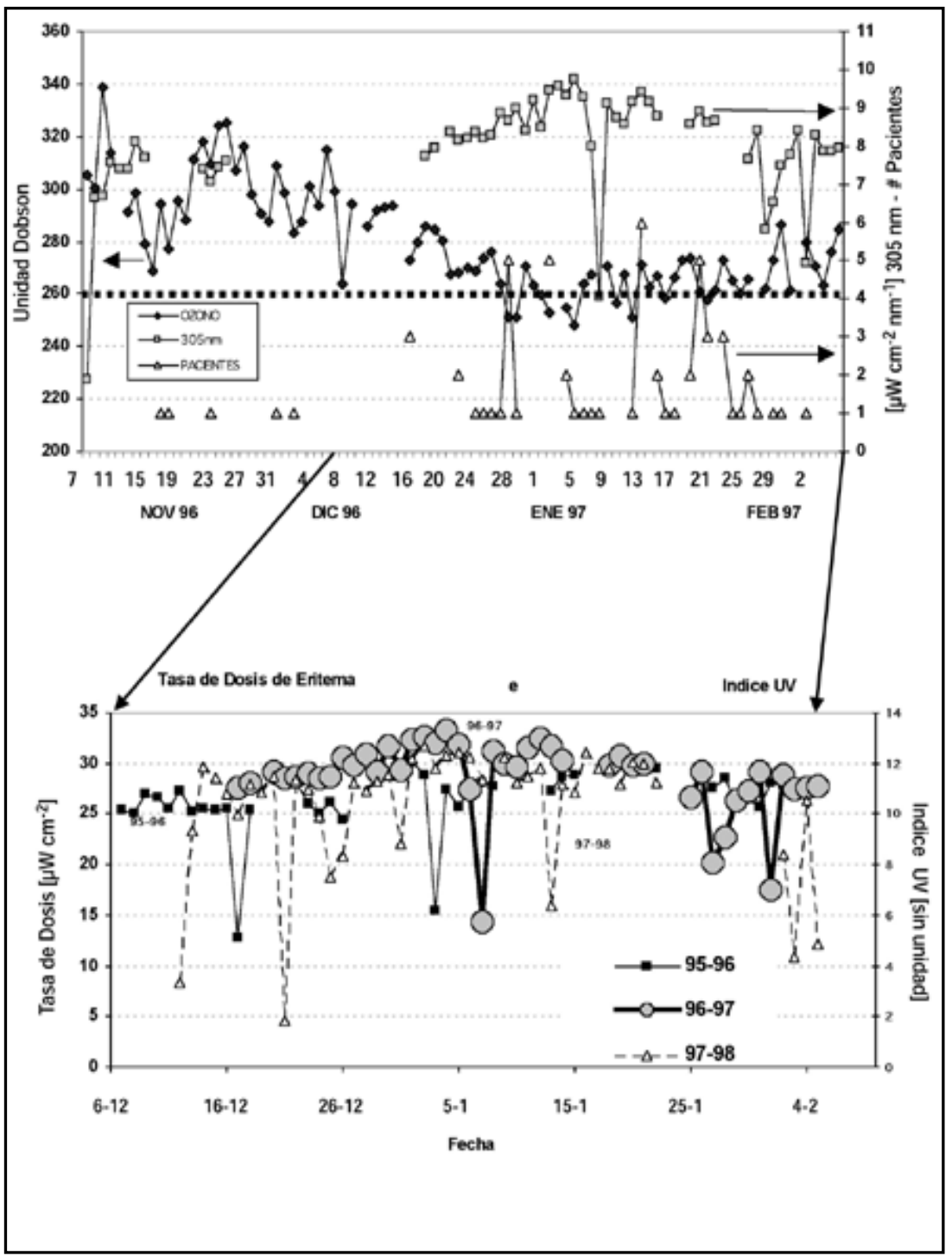

En la Figura 6, las diferencias entre los máximos valores de DDE de los veranos 95-96; 96-97 y 97-98, son pequeñas y fluctúan entre los $6.000 \mathrm{y}$ $7.000 \mathrm{~J} / \mathrm{m}^{2}$, pero son indicativas que durante el mediodía solar los índices UV y TD superaron el

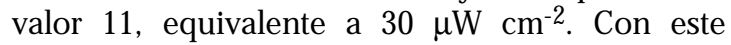
valor, en sólo $10 \mathrm{~min}$ de exposición al sol se puede provocar daño, especialmente a niños. A fines de diciembre de 1996, la DDE fluctuó entre 6.650 y $7.000 \mathrm{~J} / \mathrm{m}^{2}$ y en los 5 primeros días de enero de 1997 fueron aún superiores $(7.150 \mathrm{y}$
$7.600 \mathrm{~J} / \mathrm{m}^{2}$ ), oportunidad en que se registraron los menores valores de $\mathrm{O}_{3}$ (Figura 5a).

\section{DISCUSIÓN}

I. Consideraciones clínicas. La piel infantil, es un órgano que se encuentra en proceso de maduración, por lo cual aún no ha desarrollado completamente los mecanismos de adaptación y protección. Su función de barrera es lábil, por lo 


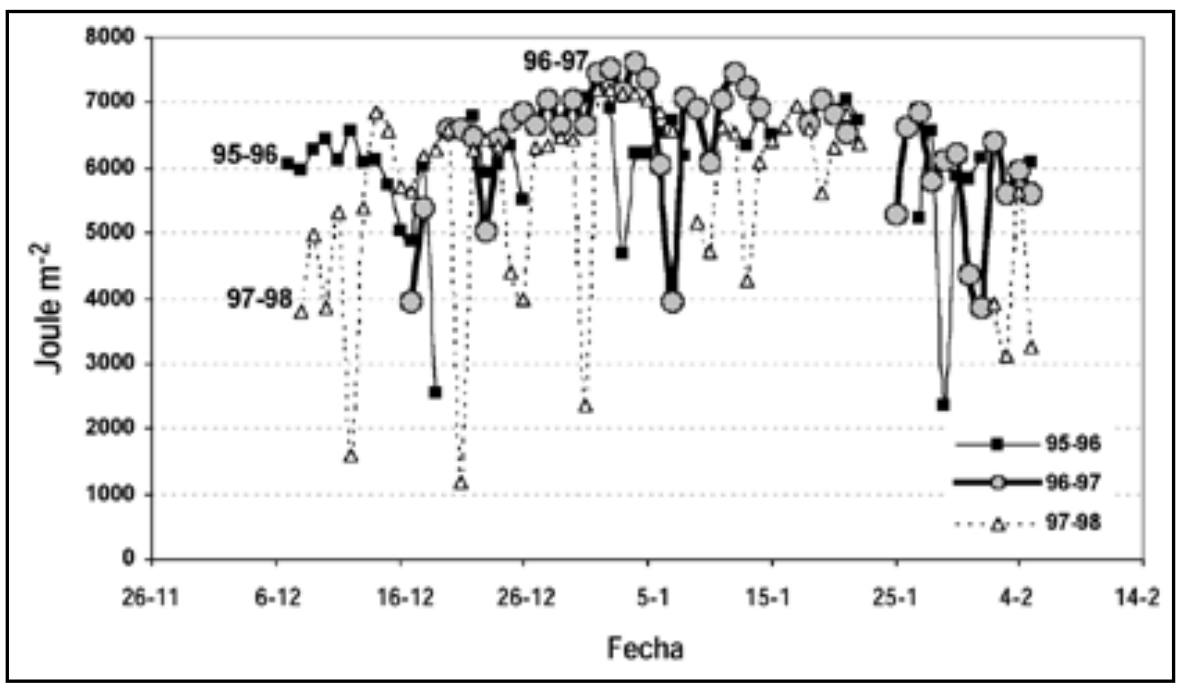

Figura 6. DDE, durante el verano de 96-97 comparado con el verano precedente y posterior. La línea negra gruesa con círculos grises representa las DDE registrados en el verano 96-97; la línea continua con cuadrados negro representa los valores DDE del verano 95-96 y la línea segmentada con triángulos abiertos representa los valores DDE del verano 97-98.

que está más expuesta a lesiones traumáticas. Estas características hacen que los niños sean más vulnerables a la radiación UV.

¿Es posible explicar la causa que produjo un mayor número de niños quemados (63) en el verano de 96-97? Cabe destacar que el número de quemados por sol, resultó independiente del número de quemados por otros agentes; también se observa que los quemados/1.000 consultantes en estas dos oportunidades se duplicaron (39 y 37 quemados/ 1.000 consultantes, 96-97 y 99-00 respectivamente) comparados con 20/1.000 consultantes o menos en los otros veranos. El número de quemados por sol en 96-97 y 99-00 son mayores, debido probablemente al incremento de UV-B provocado por un mayor número de días con bajo $\mathrm{O}_{3}$.

¿Por qué es mayor el número de niños quemados por sol que el número de niñas? Es posible que los niños jueguen más expuestos al sol, como también pueden ser más independientes y usan un traje de baño que les cubre menor superficie corporal que a las niñas. Pero también el carcinoma baso y espino celular, además del melanoma, es más frecuente en varones que en damas ${ }^{8-12}$.
El grupo etario de 6 a 10 años tiene mayor porcentaje de niños quemados, es posible que se deba a su personalidad más independiente, escapándose del control paterno. Llama mucho la atención que haya menores de un año quemados por sol.

II. Una posible explicación. Debido a que la mayoría de los quemados se presentaron en días de $\mathrm{O}_{3}$ $\leq 260$ UD, la regresión lineal entre número de días con bajo $\mathrm{O}_{3}$ y el número de quemados presenta un coeficiente de correlación significativo (Figura 7), pero lo breve del período analizado (4 años) no permite hacer inferencias concluyentes.

Durante los dos veranos críticos por el elevado número de días con bajo $\mathrm{O}_{3}$, los niños quemados se presentaron en grupos importantes: en el 96-97 fue 1 grupo de 6 niños, 3 grupos de 5 y 3 grupos de 3; en el 99-00 fue un grupo de 9, un grupo de 6 , dos grupos de 3 y 6 grupos de 2 niños. En los otros veranos, el número de niños por grupo fue menor, aunque existen grupos de 2 niños por 7 veces. Estos antecedentes sugieren una relación entre el número de días con bajo $\mathrm{O}_{3}(\leq 260 \mathrm{UD})$ y 


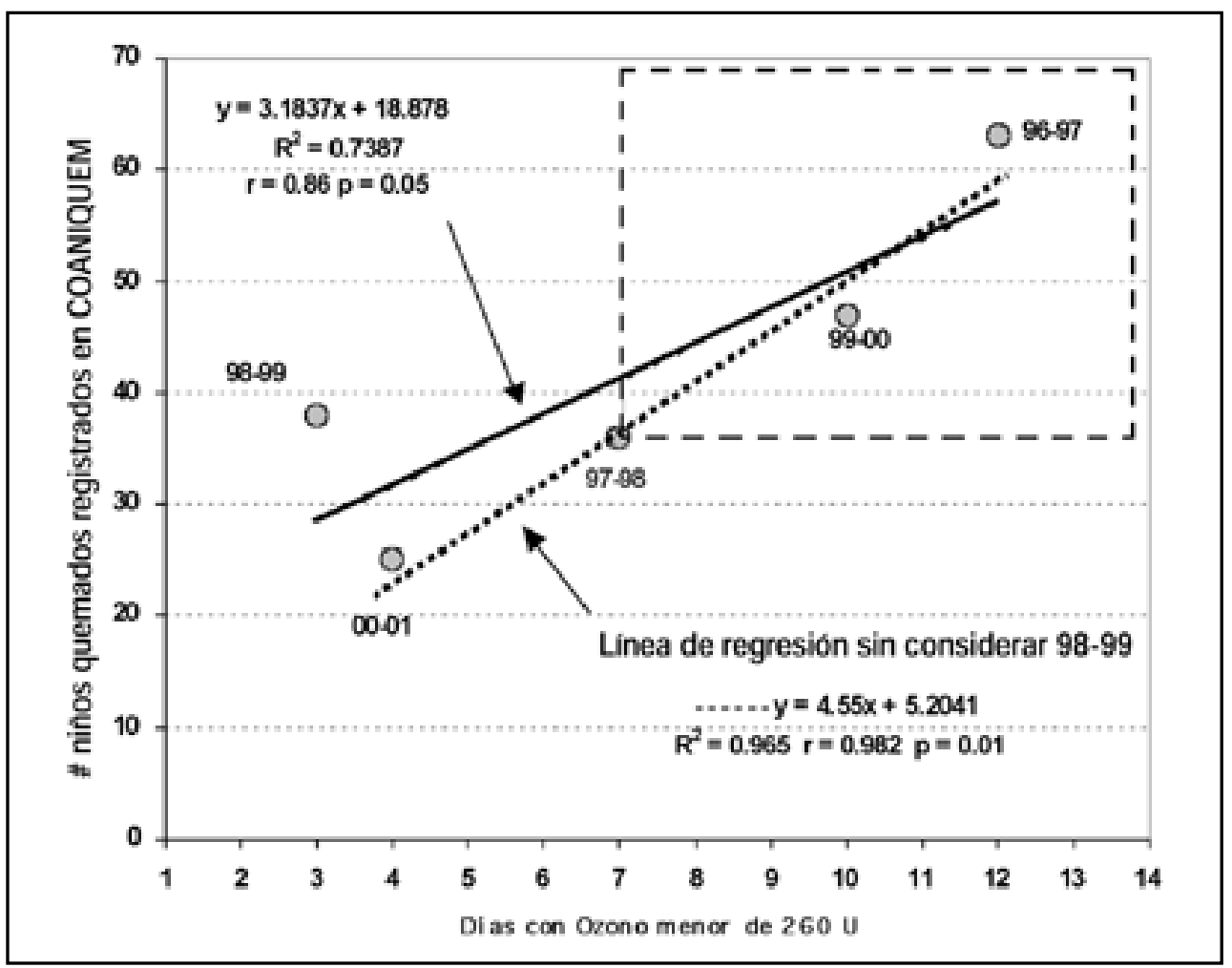

Figura 7. Número de niños quemados (eje vertical) versus número de día con valores de ozono $\leq 260$ UD (eje horizontal). La línea de regresión que incluye el verano 98-99 (línea continua); línea de regresión que excluye el verano 98-99 (línea segmentada). Verano 95-96 no tiene registros de ozono por satélite.

elevada UV-B con el mayor número de niños quemados por sol. Lo que debe considerarse como indicio de causalidad, entre cambios geofísicos y la salud de la población.

Por otra parte, el verano 00-01 fue el que presentó el menor número de días (4) con bajo $\mathrm{O}_{3}$ y también uno de los menores números de niños quemados por sol (Figura 7). El verano 9899 tuvo 38 quemados con 3 días de $\mathrm{O}_{3} \leq 260 \mathrm{UD}$, pero tuvo 22 días sin registros de $\mathrm{O}_{3}$, lo que permitiría subir a los 7,2 que completa un número más próximo a la línea de regresión, pero la incerteza es muy grande.

Con la misma ecuación para el verano 01-02 en que se registraron 5 días con $\mathrm{O}_{3} \leq 260$ UD (no se presentan estos datos), debieran haberse registrado 27 niños quemados. La página web de COANIQUEM informó que entre noviembre de 2001 y marzo de 2002 se registraron 34 quemados por sol. Estos datos aún no están disponibles. El verano 02-03 tuvo 19 días con $\mathrm{O}_{3} \leq 260 \mathrm{UD}$, lo que implicaría que deberían haberse presentado entre 80 y 90 quemados por sol, dependiendo de la ecuación que se utilice $(\mathrm{n}=50 \mathrm{n}=4)$. Cifra elevada, pero hay que esperar los registros. Por otra parte, también es posible que las campañas masivas especialmente noticiarios, pudieran estar dando resultados.

En general, cuando las bajas de $\mathrm{O}_{3}$ se han presentado en días de fin de semana, el número de casos de quemados aumenta, debido a que son las oportunidades en que padres concurren con sus hijos a piscinas u otros lugares al aire libre, donde reciben mayor radiación solar.

III. Verano 96-97. Este verano presentó el máximo de UV-B igual a $9,74 \mu \mathrm{W} \mathrm{cm}{ }^{-2} \mathrm{~nm}^{-1}$, la máxima TD

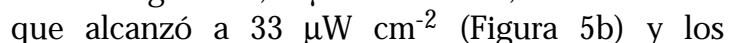
máximos índices UV. Entre el 20 de diciembre 1996 y el 20 de enero 1997 (excepto el 7 de 
enero), los índices UV fueron superiores a $10 \mathrm{y}$ en la mayoría de los casos mayores de 12 (Figura 5b), por lo cual se debió haber extremado las medidas de precaución y protección de la piel, especialmente en los menores de edad. También, se observó el mayor valor de DDE de esta serie de tiempo igual a $7.500 \mathrm{Joule} / \mathrm{m}^{2}$. En relación al verano 99-00 no tenemos la información, debido a que los valores UV no están estandarizados aún.

IV. Disminución global del $\mathrm{O}_{3}$ y aumento de UV-B. En los últimos 30 años el $\mathrm{O}_{3}$ ha disminuido progresivamente, como lo demuestran las determinaciones satelitales ${ }^{13}$. Los valores de los veranos aquí presentados (Figura 3) son todos estadísticamente diferentes al promedio histórico, a pesar que la década 79-88 ya estaba perturbada, con respecto al período anterior al agujero de ozono (1970). Herman y col ${ }^{14}$ infirieron el aumento de radiación UV dependiendo de las disminuciones de $\mathrm{O}_{3}$. Afirman que debido a que el $\mathrm{O}_{3}$ absorbe inversamente a la longitud de onda, las mediciones deben ser muy precisas. En la franja latitudinal $45^{\circ} \pm 5^{\circ} \mathrm{S}$, el promedio zonal de aumento decenal de radiación ultravioleta fue: $13 \%, 3 \%$ y $1 \%$ para las longitudes de onda de: $300 \mathrm{~nm}, 310$ $\mathrm{nm}$ y $320 \mathrm{~nm}$, respectivamente. Los promedios de verano de radiación UV-B aquí presentados no son comparables con los promedios de franja y sus variaciones ${ }^{14}$.

El valor promedio estacional de $\mathrm{O}_{3}$, parece ser menos sensible que el de la radiación UV-B; el incremento de UV-B tiene una relación lineal inversa sólo cuando la disminución de $\mathrm{O}_{3}$ es inferior a $10 \%$, pero cuando ésta es superior a $15 \%$, la relación es potencial ${ }^{15}$.

¿Cómo explicar la disminución de $\mathrm{O}_{3}$ el verano 96-97? Connor y col, en Lauder, Nueva Zelanda $\left(45^{\circ} \mathrm{S}, 170^{\circ} \mathrm{E}\right)^{16}$ mostraron que 1997 fue un año "anómalo" en $\mathrm{O}_{3}$ explicado por la Oscilación Quasi Bienal (QBO) y estimaron que la radiación UV biológicamente activa aumentó en 20 y 40\%, respecto de los años posteriores.

Sólo en el verano 96-97 la QBO presentó la fase apropiada (H Fuenzalida, comunicación personal). Dos procesos indujeron movimientos ascendentes de aire hacia la estratosfera baja y con ello empobrecieron la columna de $\mathrm{O}_{3}$. Por una parte, la QBO cuyos vientos zonales ecuatoriales de la estratosfera se encontraban en la fase propicia (cambio de vientos del este a vientos del oeste) para que la circulación meridional asociada produjera ascenso en la estratosfera baja subtropical $\left(30^{\circ} \mathrm{S}\right)$. A esto debe sumarse la dominancia de anomalías anticiclónicas (altas presiones) en la tropósfera alta de 1,5 $\sigma$ en promedio mensual sobre Santiago, lo que también se asocia con movimiento de ascenso en niveles estratosféricos. La QBO genera variaciones de 5 a 10 UD y los sistemas del tiempo unas $20 \mathrm{UD}$, las que sumadas pueden dar cuenta de la deficiencia total de $\mathrm{O}_{3}$ observada en enero de 1997.

Otros procesos que pueden estar actuando son el "Efecto de Dilución" resultado de la influencia de masas de aire pobre en $\mathrm{O}_{3}$, provenientes de la Antártica, cuando se rompe el vórtice polarr ${ }^{17}, \mathrm{u}$ "ondas gravitacionales"18. No tenemos explicación para la periodicidad trienal observada en 96-97; 9900 y 02-03 con un mayor número de días de $\mathrm{O}_{3}$ $\leq 260$ UD durante el verano. Las variables con mayor probabilidad de participación son las ondas gravitacionales. Estas le proporcionan la fuerza (torque) necesaria a la QBO cuando se producen, también influyeron en la ruptura temprana del remolino polar antártico en la primavera del año 2002. Todos estos procesos globales deben actuar individual 0 sinérgicamente para producir lo observado el verano 96-97 y en las otras oportunidades. Es indispensable continuar estudiando sus dependencias.

V. Imágenes regionales de $\mathrm{O}_{3}$ satelital. La Figura 8 representa la cobertura de $\mathrm{O}_{3}$ del país en 4 días con $\mathrm{O}_{3} \leq 260$ UD y alto número de quemados en el verano 96-97, en Santiago. Esta información sugiere que el fenómeno también se presenta en el norte, y las zonas con bajo $\mathrm{O}_{3}$ siguen la línea de la cordillera de Los Andes. Cualquiera sea la explicación, lo importante es que lo observado en Santiago podría estar simultáneamente ocurriendo en La Serena, Antofagasta o Iquique.

No tenemos una explicación al fenómeno, lo importante es continuar buscando la respuesta que permita entender el o los mecanismos que gatillan estos eventos de bajo $\mathrm{O}_{3}$ durante los veranos, para mejorar las campañas de fotoprotección. 


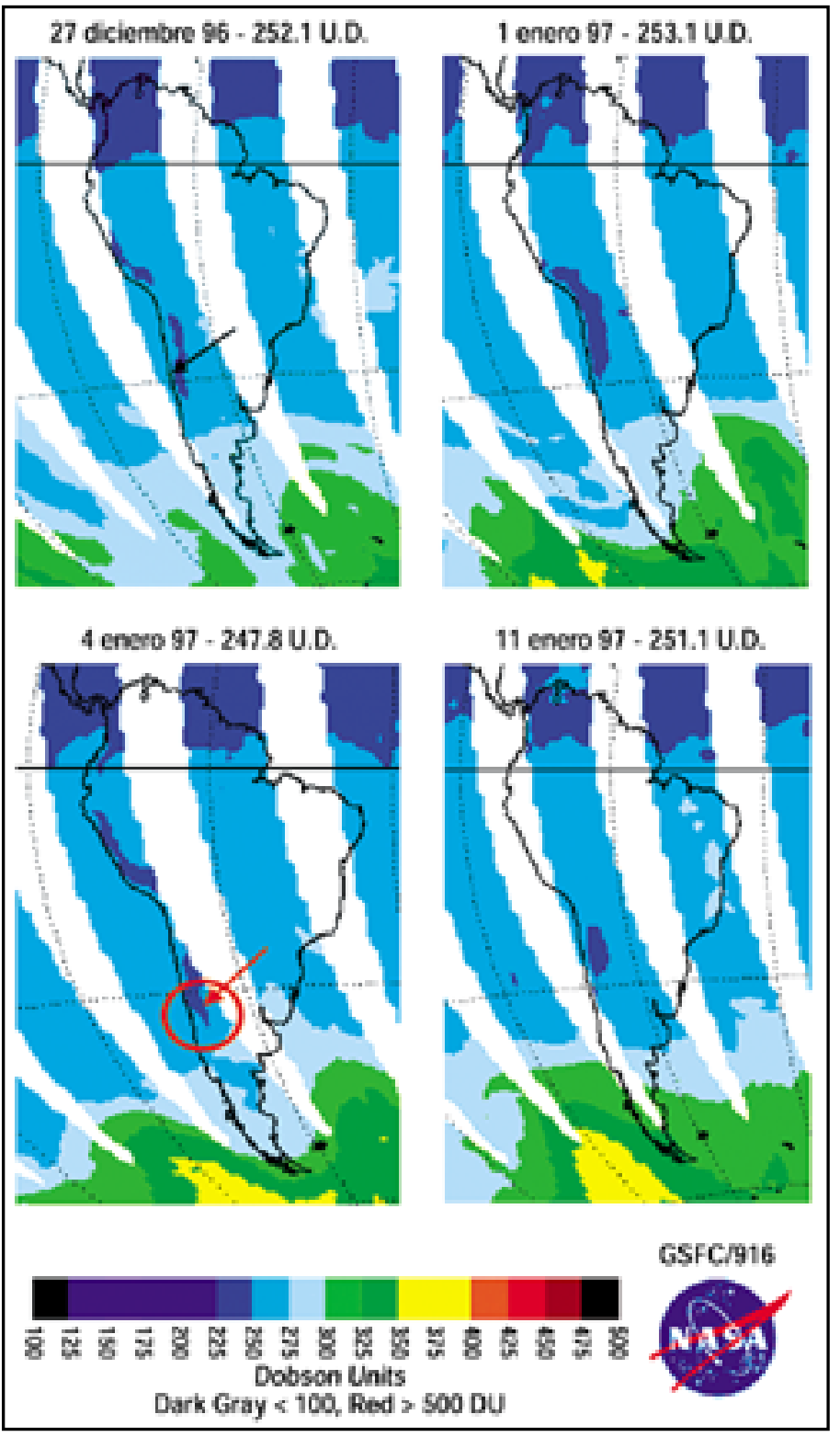

Figura 8. Imágenes satelitales de América del Sur (TOMS formato TIF) del verano 96-97. Los diferentes colores representan el grosor de la cubierta de ozono según la escala de colores. En la parte superior de la imagen se indica la fecha y el grosor de la capa de ozono sobre Santiago. La línea continua horizontal representa al Ecuador, $0^{\circ}$ y la segmentada al paralelo de latitud, $30^{\circ} \mathrm{S}$. Las manchas blancas, corresponden a zonas geográficas que quedan bajo el horizonte y el equipo TOMS no puede realizar mediciones.

\section{CONCLUSIONES}

1. Existe sincronía en la disminución de $\mathrm{O}_{3}$ estratosférico que provoca un aumento en la radiación UV-B solar y el número de niños menores de 15 años que solicitan atención en COANIQUEM por quemadura solar, en verano. Esta secuencia de hechos podría ser indicativo de causalidad.
2. Para Santiago en enero, una cubierta de $\mathrm{O}_{3}$ $\leq 260$ UD parece ser un "umbral" indicador de consecuencias en la salud de la piel expuesta a la radiación solar.

3. Las observaciones satelitales de eventos de menor cubierta de $\mathrm{O}_{3}$ que afectan a Santiago se extienden generalmente hacia o desde el norte, lo que debería mantener atenta a la población. 
4. Las medidas de fotoprotección hacia la población deben acrecentarse debido a las consecuencias de la sobreexposición y del carácter acumulativo del daño por radiación UV.

\section{REFERENCIAS}

1. WMO (World Meteorological Organization) and UNEP (United Nations Environment Programme) report: Scientific Assessment of Stratospheric Ozone: 1998. In Global Ozone Research and Monitoring Project, Report número 44, Geneve 1998.

2. Cabrera S, Fuenzalida $H$. Interannual variations of global UV radiation in Santiago. Geophys Res Lett 1999; 26: 2945-8.

3. McKinlay AF, DiFFEY BL A reference action spectrum for ultraviolet induced erythema in human skin, in: WR Passchier, BFM Bosnjakovic (Eds.), Human Exposure to Ultraviolet Radiation: Risks and Regulations, Elsevier, Amsterdam, 1987.

4. Madronich S, Mckenzie RL, Bjorn LO, CaLDWEL MM. Changes in biologically active radiation reaching the Earth's surface. J Photochem Photobiol 1998; 46: 5-19.

5. Aranibar L RUV y quemadura solar. Tesis para optar a la especialidad médica de Dermatología y Venerología. Escuela de Post Grado de la Facultad de Medicina de la Universidad de Chile. 1998.

6. FuenzaLdDA H. Global ultraviolet spectra derived directly from observations with multichannel radiometer. Applied Optic 1998; 37: 4051-61.

7. MADRONICH S. UV radiation in the natural and perturbed atmosphere. In UV-B Radiation and Ozone depletion Effects on Humans, Animals, Plants, Microorganisms, and Materials. Ed. Manfred Tevini 1993 Boca Ratón 248 pp.

8. Fears TR, Scotto J. Changes in skin cancer morbidity between 1971-72 and 1977-78. J Natl Cancer Inst 1982; 69: 365.

9. Thомas B, FitzPatrick J. Quemaduras, manejo y evaluación del paciente quemado. En: Fitzpatrick T, Eisen A, Wolff K, Freedberg I, Austen K. Dermatology in General Medicine, 4 ${ }^{\text {a }}$ Ed, New York, 1993; 1665-81.

10. Kricker A, Armstrong BK, English DR, HeEnan PJ. A dose response curve for sun exposure and basal carcinoma. Int J Cancer 1995; 60: 482-8.

11. Chuang TY, Popescu NA, Su WP, Chute CG. Scuamuos cell carcinoma. A population-based
5. Es necesario iniciar o mantener las mediciones de radiación UV-B, grosor de la cubierta de $\mathrm{O}_{3}$ y un confiable registro del número y tipo de quemaduras solares en las poblaciones de riesgo de las diferentes ciudades del país.

incidence study in Rochester, Minnesota. Arch Dermatol 1990; 126: 185-98.

12. Zemelman V, Garmendia ML, Kirschbaum A. Malignant melanoma mortality rates in Chile (1988-98). Int J Dermatol 2002; 41: 99-102.

13. Bojkov RD, Fioletov VE. Estimating the global ozone characteristic during the last 30 years. J Geophys Res 1995; 100: 16537-51.

14. Herman JR, Bhartia PK, Ziemke J, Ahmad Z, Larko D. UV-B increases (1979-1992) from decreases in total ozone. Geophys Res Lett 1996; 23: 2117-20.

15. BоотH CHR, MADRONICH S. Radiation amplification factors: Improved formulation accounts for large increases in ultraviolet radiation associated with Antarctic ozone depletion. Antarct Res Ser 1994; 62: 39-42.

16. Connor BJ, Bodeker GE, Mckenzie RL, Boyd IS. The total ozone anomaly at Lauder, NZ in 1997. Geophys Res Lett 1999; 26: 189-92.

17. Roy CT, Gies HP, Graime E. Ozone depletion. Science 1990; 347: 235-6.

18. AIEXANDER MJ, HoLton JR A model study of zonal forcing in the equatorial stratosphere by convectively induced gravity waves. J Atmos Sci 1997; 54: 408-19.

\section{Agradecimientos}

Los autores agradecen a COANIQUEM (http:// www.coaniquem.cl) por las facilidades otorgadas para desarrollar este trabajo, así como también al Proyecto IAI "InterAmerican Institute" Global Change Research CNR 026 y al Departamento de Investigación y Desarrollo (DID), de la Universidad de Chile por colaborar parcialmente en el desarrollo de esta investigación. Especial mención deseamos hacer para la National Aeronautic and Space Administration (NASA, cuya página Web es: http:// jwocky.gsfc.nasa.gov/), por permitir a la comunidad científica acceder a la información que ellos producen, procesan y diariamente publican. Deseamos agradecer a la Dra. María Vernet (Scripps Institution of Oceanography, La Jolla. CA) y a tres revisores anónimos por sus valiosos comentarios y sugerencias que mejoraron el manuscrito. 